Research Article

\title{
A note on rainbow mean indexes of paths
}

\author{
Gary Chartrand ${ }^{1}$, James Hallas ${ }^{1}$, Ebrahim Salehi ${ }^{2}$, Ping Zhang ${ }^{1, *}$ \\ ${ }^{1}$ Department of Mathematics, Western Michigan University, Kalamazoo, Michigan 49008-5248, USA \\ ${ }^{2}$ Department of Mathematical Sciences, University of Nevada Las Vegas, Las Vegas, Nevada 89154-4020, USA
}

(Received: 28 September 2021. Accepted: 25 October 2021. Published online: 29 October 2021.)

(C) 2021 the authors. This is an open access article under the CC BY (International 4.0) license (www.creativecommons.org/licenses/by/4.0/).

\begin{abstract}
For an edge coloring $c$ of a connected graph $G$ of order 3 or more with positive integers, the chromatic mean of a vertex $v$ of $G$ is defined as that vertex color which is the average of the colors of the edges incident with $v$. Only those edge colorings $c$ for which the chromatic mean of every vertex is a positive integer are considered. If distinct vertices have distinct chromatic means, then $c$ is called a rainbow mean coloring of $G$. The maximum vertex color in a rainbow mean coloring $c$ of $G$ is the rainbow mean index of $c$, while the rainbow mean index of $G$ is the minimum rainbow mean index among all rainbow mean colorings of $G$. In this note, we prove that every path $P_{n}$ of order $n \geq 3$ has rainbow mean index $n$ except $P_{4}$ which has rainbow mean index 5 .
\end{abstract}

Keywords: chromatic mean; rainbow mean colorings; rainbow mean index; path.

2020 Mathematics Subject Classification: 05C07, 05C15, $05 \mathrm{C} 78$.

\section{Introduction}

An edge coloring $c$ of a connected graph $G$ of order 3 or more with positive integers is called a mean coloring of $G$ if the chromatic mean $\mathrm{cm}(v)$ of each vertex $v$ of $G$, defined by

$$
\operatorname{cm}(v)=\frac{\sum_{e \in E_{v}} c(e)}{\operatorname{deg} v}, \text { where } E_{v} \text { is the set of edges incident with } v,
$$

is an integer. If distinct vertices have distinct chromatic means, then the edge coloring $c$ is called a rainbow mean coloring of $G$. This concept was introduced and studied in [2] and more information on this topic has been presented in [1,3]. It was shown in [2] that every connected graph of order 3 or more has a rainbow mean coloring.

For a rainbow mean coloring $c$ of a connected graph $G$ of order 3 or more, the maximum vertex color is the rainbow chromatic mean index (or simply, the rainbow mean index) $\mathrm{rm}(c)$ of $c$. That is,

$$
\operatorname{rm}(c)=\max \{\operatorname{cm}(v): v \in V(G)\} .
$$

The rainbow chromatic mean index (or the rainbow mean index) $\operatorname{rm}(G)$ of the graph $G$ itself is defined as

$$
\operatorname{rm}(G)=\min \{\operatorname{rm}(c): \quad c \text { is a rainbow mean coloring of } G\} .
$$

Consequently, if $G$ is a connected graph of order $n \geq 3$, then $\operatorname{rm}(G) \geq n$.

It was stated in [2] that every path $P_{n}$ of order $n \geq 3$ has rainbow mean index $n$ except for $P_{4}$ which has rainbow mean index 5. Because of the page limit of the journal, the proof of this fact was not included in [2]. The proof is given here because one of the authors recently received a note for review in which the main result of the present note was erroneously disproved. Therefore, we write this note to provide a proof of this fact.

\section{Main result}

First, we present the rainbow mean index of $P_{4}$, which appears in [2]. We include a proof of this result here for completeness.

Proposition 2.1. $\operatorname{rm}\left(P_{4}\right)=5$.

Proof. The edge coloring in Figure 1 shows that $\operatorname{rm}\left(P_{4}\right) \leq 5$.

\footnotetext{
*Corresponding author (ping.zhang@wmich.edu).
} 


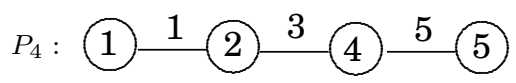

Figure 1: A rainbow mean coloring of $P_{4}$.

Next, we show that $\operatorname{rm}\left(P_{4}\right) \geq 5$. Assume, to the contrary, that there is a rainbow mean coloring $c$ of $P_{4}$ such that $\operatorname{rm}(c)=4$. Let $P_{4}=\left(v_{1}, v_{2}, v_{3}, v_{4}\right)$. Since $\left\{\mathrm{cm}\left(v_{i}\right): 1 \leq i \leq 4\right\}=[4]$, no two edges can be colored the same. Consequently, since only one vertex is colored 1 , this implies that $\operatorname{cm}\left(v_{1}\right)=1$ or $\mathrm{cm}\left(v_{4}\right)=1$. We may assume that $\mathrm{cm}\left(v_{1}\right)=1$ and so $c\left(v_{1} v_{2}\right)=1$. Hence, the edges of $P_{4}$ are colored with distinct odd integers. If some edge of $P_{4}$ is colored 7 or more, then some vertex of $P_{4}$ is colored 5 or more, which is impossible. Consequently, $\left\{c\left(v_{i} v_{i+1}\right): i=1,2,3\right\}=\{1,3,5\}$ and so $\left\{c\left(v_{2} v_{3}\right), c\left(v_{3} v_{4}\right)\right\}=\{3,5\}$. In either case, it follows that $\left\{\operatorname{cm}\left(v_{i}\right): 1 \leq i \leq 4\right\} \neq[4]$, which is a contradiction. Therefore, $\operatorname{rm}\left(P_{4}\right) \geq 5$ and so $\operatorname{rm}\left(P_{4}\right)=5$.

By Proposition 2.1, if $n=4$, then $\operatorname{rm}\left(P_{n}\right)=n+1$. Next, we show that $P_{4}$ is the only exception for all paths $P_{n}$ of order $n \geq 3$.

Theorem 2.1. For each integer $n \geq 3$ and $n \neq 4, \operatorname{rm}\left(P_{n}\right)=n$.

Proof. Since $\operatorname{rm}\left(P_{n}\right) \geq n$ for all integers $n \geq 3$, it remains to show that there is a rainbow mean coloring $c$ of $P_{n}$ such that $\operatorname{rm}(c)=n$. Let $P_{n}=\left(v_{1}, v_{2}, \ldots, v_{n}\right)$ and let $e_{i}=v_{i} v_{i+1}$ for $1 \leq i \leq n-1$. First, suppose that $n \geq 3$ is odd. Define the edge coloring $c: E\left(P_{n}\right) \rightarrow[n]$ of $P_{n}$ by $c(e)=i$ if $e$ is incident with $v_{i}$ where $1 \leq i \leq n$ and $i$ is odd. Figure 2 shows such an edge coloring of $P_{n}$ for $n=3,5,7$. Since $\operatorname{cm}\left(v_{i}\right)=i$ for $1 \leq i \leq n$, it follows that $c$ is a rainbow mean coloring of $P_{n}$ with $\operatorname{rm}(c)=n$. Therefore, $\operatorname{rm}\left(P_{n}\right)=n$ for each odd integer $n \geq 3$.

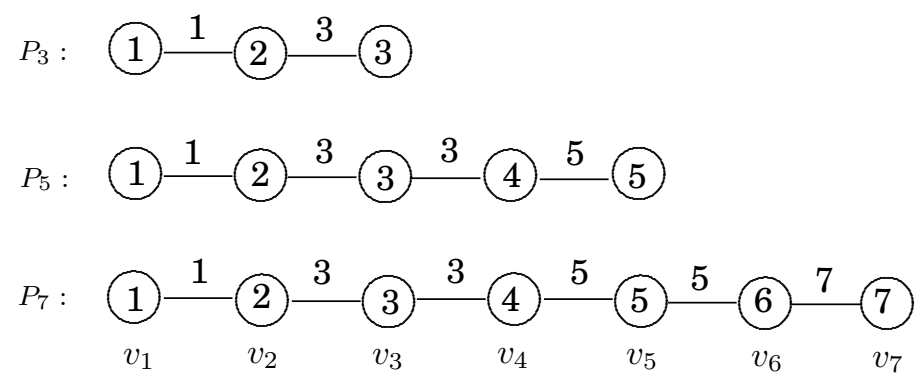

Figure 2: Rainbow mean colorings of $P_{3}, P_{5}$, and $P_{7}$.

We may therefore assume that $n \geq 6$ is even. Since $n \geq 6$ is even, it follows that either $n \equiv 2(\bmod 4)$ or $n \equiv 0(\bmod 4)$. We proceed by induction to prove the following statements.

- If $n \equiv 2(\bmod 4)$, then there exists a rainbow mean coloring $c_{n}$ of $P_{n}$ such that $c_{n}\left(e_{n-1}\right)=3$ and $\operatorname{rm}\left(c_{n}\right)=n$.

- If $n \equiv 0(\bmod 4)$ and $n \geq 8$, then there exists a rainbow mean coloring $c_{n}$ of $P_{n}$ such that $c_{n}\left(e_{n-1}\right)=5$ and $\operatorname{rm}\left(c_{n}\right)=n$.

The edge colorings of $P_{6}$ and $P_{8}$ in Figure 3 show that the statements are true for $n=6,8$. Suppose that the statement is true for an arbitrary even integer $n \geq 6$. Next, we show that the statement is true for $n+4$ by considering two cases, according to whether $n \equiv 2(\bmod 4)$ or $n \equiv 0(\bmod 4)$. We use $\mathrm{cm}_{t}(v)$ to denote the chromatic mean of a vertex $v$ with respect to an edge coloring $c_{t}$ of the path $P_{t}$ of order $t$.

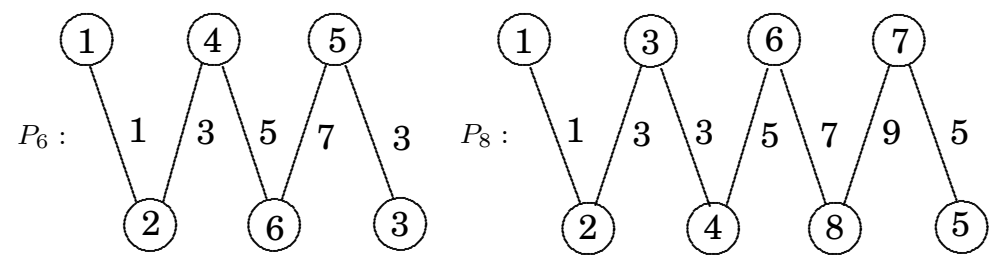

Figure 3: Rainbow mean colorings of $P_{6}$ and $P_{8}$.

Case 1. $n \equiv 2(\bmod 4)$. By the induction hypothesis, there is a rainbow mean coloring $c_{n}$ of $P_{n}$ such that $c_{n}\left(e_{n-1}\right)=$ $\mathrm{cm}_{n}\left(v_{n}\right)=3$ and $\left\{\mathrm{cm}_{n}\left(v_{i}\right): 1 \leq i \leq n\right\}=[n]$. We now extend $c_{n}$ to an edge coloring $c_{n+4}$ of $P_{n+4}$ by defining $c_{n+4}\left(e_{n}\right)=2 n+1$, $c_{n+4}\left(e_{n+1}\right)=1, c_{n+4}\left(e_{n+2}\right)=2 n+5$, and $c_{n+4}\left(e_{n+3}\right)=3$. Then $\mathrm{cm}_{n+4}\left(v_{i}\right)=\mathrm{cm}_{n}\left(v_{i}\right)$ for $1 \leq i \leq n-1$ and $\mathrm{cm}_{n+4}\left(v_{n}\right)=n+2$, $\mathrm{cm}_{n+4}\left(v_{n+1}\right)=n+1, \mathrm{~cm}_{n+4}\left(v_{n+2}\right)=n+3, \mathrm{~cm}_{n+4}\left(v_{n+3}\right)=n+4$, and $\mathrm{cm}_{n+4}\left(v_{n+4}\right)=3$. It follows that 


$$
\left\{\mathrm{cm}_{n+4}\left(v_{i}\right): 1 \leq i \leq n+4\right\}=[n+4] .
$$

Figure 4 illustrates the construction of such an edge coloring for $n=6$, where a rainbow mean coloring $c_{10}$ of $P_{10}$ is constructed from the given rainbow mean coloring $c_{6}$ of $P_{6}$.

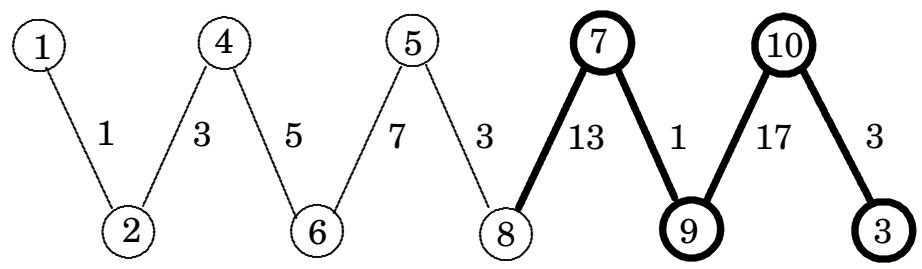

Figure 4: The construction of the rainbow mean coloring $c_{10}$ of $P_{10}$ in Case 1 in the proof of Theorem 2.1.

Case 2. $n \equiv 0(\bmod 4)$ and $n \geq 8$. By the induction hypothesis, there is a rainbow mean coloring $c_{n}$ of $P_{n}$ such that $c_{n}\left(e_{n-1}\right)=\mathrm{cm}_{n}\left(v_{n}\right)=5$ and $\left\{\mathrm{cm}_{n}\left(v_{i}\right): 1 \leq i \leq n\right\}=[n]$. We now extend $c_{n}$ to an edge coloring $c_{n+4}$ of $P_{n+4}$ by defining $c_{n+4}\left(e_{n}\right)=2 n-3, c_{n+4}\left(e_{n+1}\right)=7, c_{n+4}\left(e_{n+2}\right)=2 n+1$, and $c_{n+4}\left(e_{n+3}\right)=5$. Then $\mathrm{cm}_{n+4}\left(v_{i}\right)=\mathrm{cm}_{n}\left(v_{i}\right)$ for $1 \leq i \leq n-1$ and $\mathrm{cm}_{n+4}\left(v_{n}\right)=n+1, \mathrm{~cm}_{n+4}\left(v_{n+1}\right)=n+2, \mathrm{~cm}_{n+4}\left(v_{n+2}\right)=n+4, \mathrm{~cm}_{n+4}\left(v_{n+3}\right)=n+3$, and $\mathrm{cm}_{n+4}\left(v_{n+4}\right)=5$. Thus, $\left\{\mathrm{cm}_{n+4}\left(v_{i}\right): 1 \leq i \leq n+4\right\}=[n+4]$. Figure 5 illustrates the construction of such an edge coloring for $n=8$, where a rainbow mean coloring $c_{12}$ of $P_{12}$ is constructed from the given rainbow mean coloring $c_{8}$ of $P_{8}$.

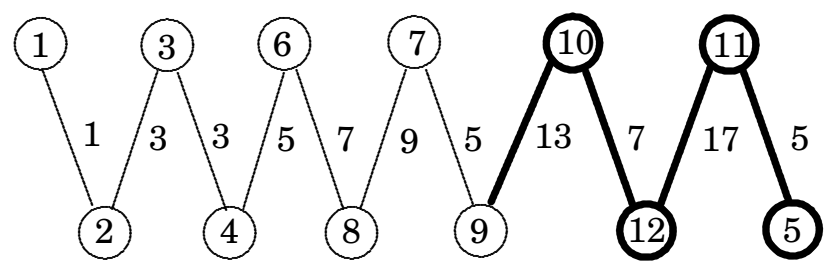

Figure 5: The construction of a rainbow mean coloring $c_{12}$ of $P_{12}$ in Case 2 in the proof of Theorem 2.1.

By Proposition 2.1 and Theorem 2.1, we have the following result.

Corollary 2.1. For each integer $n \geq 3$,

$$
\operatorname{rm}\left(P_{n}\right)=\left\{\begin{array}{cc}
n+1 & \text { if } n=4 \\
n & \text { if } n \neq 4
\end{array}\right.
$$

\section{References}

[1] A. Ali, G. Chartrand, P. Zhang, Irregularity in Graphs, Springer, New York, 2021.

[2] G. Chartrand, J. Hallas, E. Salehi, P. Zhang, Rainbow mean colorings of graphs, Discrete Math. Lett. 2 (2019) 18-25.

[3] J. Hallas, E. Salehi, P. Zhang, Rainbow mean colorings of bipartite graphs, Bull. Inst. Combin. Appl. 88 (2020) 78-97. 